\title{
Large time behaviour of fronts governed by eikonal equations
}

\author{
GUY BARLES ${ }^{\dagger}$ \\ Laboratoire de Mathématiques et Physique Théorique (UMR 6083), \\ Faculté des Sciences et Techniques, Université de Tours, \\ Parc Grandmont, 37200 Tours, France \\ AND \\ JEAN-MICHEL ROQUEJOFFRE \\ UFR-MIG, UMR CNRS 5640, \\ Université Paul Sabatier, \\ 118 route de Narbonne, 31062 Toulouse Cedex, France
}

[Received 25 May 2002 and in revised form 15 October 2002]

\begin{abstract}
Motivated by a model of solid combustion in heterogeneous media, we investigate the timeasymptotic behaviour of flame fronts evolving with a periodic space-dependent normal velocity; using the so-called "level set approach" we are led to study the large time behaviour of solutions of eikonal equations. We first provide a general approach which shows that the asymptotic normal velocity of such a flame front depends only on its normal direction and is given by the homogenized Hamiltonian of the eikonal equation. Then we turn to a more precise study of the asymptotic behaviour of the flame front when the initial front is a graph of a periodic function: in this case, the front moves asymptotically with a constant normal velocity and we are able to prove that, in coordinates moving with this constant velocity, the front has a time-periodic asymptotic behaviour in the following two cases: (i) when there is a straight line of maximal speed, and (ii) when the space dimension is 2 . These results are obtained by using homogenization, control theory and dynamical systems methods (Aubry-Mather sets).
\end{abstract}

Keywords: Front propagation; level set approach; eikonal equations; periodic solutions; homogenization; Aubry-Mather set.

\section{Introduction}

The problem under study is the large time behaviour of multi-dimensional fronts propagating under eikonal equations. The propagation law of the front reads

$$
V_{n}=R(X),
$$

where $V_{n}$ is the normal speed of the front at the point $X$ and $R: \mathbb{R}^{N} \rightarrow \mathbb{R}$ is a smooth, $\mathbb{Z}^{N}$-periodic function which is bounded from below by a positive constant. The main underlying motivation is a model of solid combustion in heterogeneous media where the flame front is assumed to propagate in $\mathbb{R}^{N}$ with a periodic, space dependent normal velocity. We refer to Namah and Roquejoffre [25] and reference therein for a more detailed presentation of the model.

\footnotetext{
Email: barles@univ-tours.fr
}

Email: roque@mip.ups-tlse.fr 
This type of models was proposed by Landau in the 40's, in particular a simpler example when $R$ is a constant: even in this case, it is known that fronts which are smooth for all time do not exist in general, and in Barles [3], a weak formulation to study the simple Landau model was proposed; it was based on the idea that the moving front can be identified as the 0-level set of the unique viscosity solution of an eikonal equation. This kind of idea was first used for numerical computations by Osher and Sethian [27] who extended it to more general normal velocities (in particular curvature dependent ones). Then Evans and Spruck [16] and Chen, Giga and Goto [12] developed the theoretical basis of the so-called "level set approach".

In our case, the level set approach for 1.1 consists in solving the eikonal equation

$$
u_{t}+R(x)|D u|=0 \quad \text { in }(0,+\infty) \times \mathbb{R}^{N},
$$

the flame front at time $t$, denoted by $\Gamma_{t}$, being identified as the 0-level set of $u(t, \cdot)$.

Equation 1.2 generates a nonlinear semigroup denoted by $\mathcal{S}(t)$ and below we also use the fact that the solution of the Cauchy problem for 1.2 is given by an explicit formula coming from control theory, namely

$$
u(t, x)=\inf _{\gamma(t)=x,|\dot{\gamma}| \leqslant R(\gamma)} u(0, \gamma(0))
$$

where $\gamma$ is taken among all piecewise $C^{1}$ curves.

In this framework, the problem of studying the asymptotic behaviour of the flame front $\Gamma_{t}$ leads to the investigation of the large time behaviour of the nonlinear semigroup $\mathcal{S}(t)$ and/or of the geodesics $\gamma$ in 1.3 and we will address this question under various assumptions on the initial datum $u(0, \cdot)$.

In two space dimensions, and when the function $R$ is constant along parallel lines, the problem is treated in [25] where two results are obtained.

- For any $p \in \mathbb{R}^{2}$-not necessarily parallel to the lines along which $R$ is constant-there is a unique $V_{p}>0$ such that $(1.2)$ has solutions of the form $p \cdot x-V_{p} t+\phi\left(p^{*} \cdot x\right)$, where $p^{*}$ is any vector orthogonal to $p$. Hence, for flat initial front, there is an asymptotic velocity $V_{p}$.

- If the front is forced to propagate in direction $p$ or, in other words, if the initial datum $u_{0}$ is such that

$$
\lim _{p \cdot x \rightarrow \pm \infty} u_{0}(x)= \pm 1
$$

and is periodic in the direction $p^{*}$ orthogonal to $p$, then $\mathcal{S}(t) u_{0}\left(p \cdot x-V_{p} t, p^{*} \cdot x\right)$ converges to a time-periodic solution; therefore, moving at velocity $V_{p}$, an observer would see the front having a periodic behaviour. Technically, this problem could basically be solved by reduction to an essentially 1-D problem, where explicit computations are tractable.

The aim of this paper is to examine what can be said in a greater generality, first about the asymptotic normal velocity of $\Gamma_{t}$ and then on a more precise behaviour, which can be viewed as looking at a second term in an expansion.

Using homogenization theory, and in particular Lions, Papanicolaou and Varadhan's [23] results, we first show that the asymptotic normal velocity is $\bar{H}(n)$ where $n$ stands for the normal direction to the front and $\bar{H}$ is the homogenized Hamiltonian associated to the Hamiltonian $H(x, p):=$ $R(x)|p|$. This result can be viewed as the analogue of the propagation results in Aronson and Weinberger [1] for the nonlinear diffusion equation $u_{t}-\Delta u=(u+1)(1-u)$ : the front $\{u=0\}$ is shown to move — at least for initial data that are equal to -1 outside a compact set —as the solution 
of $u_{t}+c^{*}|D u|=0$, where $c^{*}$ is the minimal speed of the travelling waves solutions of the 1-D equation.

When the initial front is a hyperplane, namely $\Gamma_{0}=\{x: p \cdot x=0\}$ with $p \in \mathbb{R}^{N}$, or a bounded perturbation of it, this result implies that $\Gamma_{t}$ looks like $\bar{\Gamma}_{t}=\{x: p \cdot x=t \bar{H}(p)\}$ for large $t$ and the next step consists in studying the behaviour of $\Gamma_{t}$ in coordinates moving with $\bar{\Gamma}_{t}$, which typically leads to consider the asymptotic behaviour of $u(t, \cdot)-t \bar{H}(p)$ where $u$ is the solution of 1.2 with initial data $u(0, x)=p \cdot x+v_{0}(x), v_{0}$ being a continuous, $\mathbb{Z}^{N}$-periodic function. With this formulation, it is clear that we look for the second term in the asymptotic expansion of $u(t, x)$. It is worth pointing out anyway that, in Section 4, we use a little bit more complicated (but natural) change of the $u$ variable in order to obtain a more precise behaviour of the front and that we have not been able to do it differently.

We are able to prove the convergence of $u(t, \cdot)-t \bar{H}(p)$ to a space-time periodic function in two cases: (i) when there are lines of maximum velocity (corresponding to lines of maximal value for $R$ ) and the hyperplane is orthogonal to these lines, and (ii) when the space dimension is 2 . We point out that the information is obtained on the function $u$ itself (or on suitable functions obtained by changing the $u$ variable, e.g. $u(t, \cdot)-t \bar{H}(p))$ and not on its 0 -level set: indeed, we do not know whether the empty interior property is satisfied asymptotically in time, even if it is satisfied at time $t=0$.

We conclude this introduction by providing few additional references on the study of the asymptotic behaviour of solutions of Hamilton-Jacobi equations and homogenization, which are the two main themes here. The first results on the convergence of the Oleinnik-Lax semigroup-the analogue of the $\mathcal{S}$ above-in the case when the limiting behaviour was the convergence to a solution of the stationary equation, were obtained by Namah and the second author [26]: under a certain structure condition of the Hamiltonian, they were able to prove such a result by mixing the classical half-relaxed limit method and monotonicity properties of the solution of the evolution equation on some subset of $\mathbb{R}^{N}$. The complete result for convex, superlinear and smooth Hamiltonians was obtained by Fathi [17, 18, 19], using dynamical system methods: in this series of works, Fathi shows the importance of the Aubry-Mather set and provides several very interesting properties of the solutions of the related Hamilton-Jacobi equations. A PDE proof of Fathi's result, and even of an extension to not necessarily convex nor smooth Hamiltonians, was given in Barles and Souganidis [6] who also show in [7] that several assumptions, though apparently restrictive, are necessary to have such asymptotic behaviour.

Not so many results exist on the convergence to time-periodic solutions: one reason for that is the counter-example of Fathi and Mather [20] (see also Barles and Souganidis [7] for a simpler, explicit example) showing that even for a strictly convex, smooth time-periodic Hamiltonian, the convergence may fail. The second author [28] provides such results but, and this is a key difference with the present work, he assumes the Hamiltonian to be strictly convex and time-periodic (none of these assumptions is satisfied here) and proves the convergence to solutions whose periods in time are multiples of the period of the Hamiltonian: here, at least in Section 5, the period in time is given by the asymptotic behaviour of the geodesics in 1.3 and is not a priori given in the problem. Nonetheless, in Section 5 we will use the ideas presented in [28] in a crucial way.

The recent paper of Evans and Gomes [15] connects homogenization with some features of the time-asymptotic behaviour of solutions of Hamilton-Jacobi equations, and in particular, the AubryMather set. Here we will not use such approach since the homogenization part is used only to have the "rough" asymptotic velocity and not the more precise behaviour. We also point out the work 
of Concordel [13, 14] who obtained explicit formulas for the homogenized Hamiltonian, a difficult task in general.

The paper is divided into four sections. In Section 2, we provide the general approach to the asymptotic behaviour of the front. Section 3 is devoted to explicit computations, and in particular to the connections with the approach in [25]. Section 4 is devoted to the case when there exist lines of maximal value for $R$, and Section 5 treats the 2-dimensional case.

\section{The asymptotic normal velocity}

In this section we present a general approach to the study of the asymptotic behaviour as $t \rightarrow+\infty$ of the flame front $\left(\Gamma_{t}\right)_{t}$ and in particular we show the existence of an asymptotic normal velocity.

In order to be more specific, we introduce an open subset $\Omega_{0}$ of $\mathbb{R}^{N}$ which, in terms of our solid combustion model, can be interpreted as the burnt region; the initial flame front $\Gamma_{0}$ is nothing but $\partial \Omega_{0}$ and the unburnt region is $\bar{\Omega}_{0}^{c}$. The burnt region is allowed to be either bounded or unbounded.

As is rather standard in the level set approach, we introduce the signed distance to $\Gamma_{0}$, denoted by $d\left(x, \Gamma_{0}\right)$, which is negative in $\Omega_{0}$ and positive in $\bar{\Omega}_{0}^{c}$.

We solve equation $[1.2]$ with the initial data

$$
u(0, x)=d\left(x, \Gamma_{0}\right) \quad \text { in } \mathbb{R}^{N} .
$$

Our result is

THEOREM 2.1 Under the above assumptions on $R$, there exists a function $\bar{H}: \mathbb{R}^{N} \rightarrow \mathbb{R}$ such that $\left(\Gamma_{t}\right)_{t}$ moves asymptotically as $t \rightarrow+\infty$ with normal velocity $\bar{H}(n)$ where $n$ denotes the unit normal to the front $\Gamma_{t}$ pointing in the direction of unburnt gas. More precisely:

(i) if there exists some subset $\bar{\Gamma}_{0}$ of $\mathbb{R}^{N}$ such that $d\left(\varepsilon \Gamma_{0}, \bar{\Gamma}_{0}\right) \rightarrow 0$ when $\varepsilon \downarrow 0$ with $\left\{d\left(\cdot, \bar{\Gamma}_{0}\right)<0\right\}$ nonempty and if $\left(\bar{\Gamma}_{t}\right)_{t}$ is the evolution of $\bar{\Gamma}_{0}$ with normal velocity $\bar{H}(n)$ then

$$
\frac{1}{t} d\left(\Gamma_{t}, \bar{\Gamma}_{t}\right) \rightarrow 0 \quad \text { as } t \rightarrow+\infty .
$$

(ii) If $d\left(\varepsilon \Gamma_{0}, \bar{\Gamma}_{0}\right)=O(\varepsilon)$ when $\varepsilon \downarrow 0$ then

$$
d\left(\Gamma_{t}, \bar{\Gamma}_{t}\right)=O(1) \quad \text { as } t \rightarrow+\infty .
$$

It is worth remarking that the two results (i) and (ii) of Theorem 2.1 take into account two situations that are a priori different; indeed, if $\Gamma_{0}$ is bounded, then $\bar{\Gamma}_{0}$ is nothing but $\{0\}$ and the assumption on $\left\{d\left(\cdot, \bar{\Gamma}_{0}\right)<0\right\}$ cannot be satisfied; therefore (i) does not apply at all to this bounded case. On the contrary, (ii) does apply.

If $\Gamma_{0}$ is unbounded, then both cases (i) and (ii) may apply and they do in particular if $\Gamma_{0}$ is a hyperplane (like $p \cdot x=a$ ), showing that the distance between $\Gamma_{t}$ and $\bar{\Gamma}_{t}:=\{x: p \cdot x=t \bar{H}(p)\}$ is bounded.

It is worth pointing out that, in any case, when $\bar{\Gamma}_{0}$ exists, it is a cone with vertex at the origin.

Proof of Theorem 2.1. As already mentioned, in order to study the behaviour of the moving front $\left(\Gamma_{t}\right)_{t}$, we study the behaviour of appropriate solutions of $[1.2$ as $t \rightarrow \infty$. 
First we perform a scaling $(t, x) \mapsto(t / \varepsilon, x / \varepsilon)$ and introduce the function $u^{\varepsilon}$ defined by $u^{\varepsilon}(t, x):=\varepsilon u(t / \varepsilon, x / \varepsilon)$. This new function solves

$$
\begin{aligned}
& u_{t}^{\varepsilon}+R(x / \varepsilon)\left|D u^{\varepsilon}\right|=0 \quad \text { in }(0,+\infty) \times \mathbb{R}^{N}, \\
& u^{\varepsilon}(x, 0)=\varepsilon d\left(x / \varepsilon, \Gamma_{0}\right)=d\left(x, \varepsilon \Gamma_{0}\right) \quad \text { in } \mathbb{R}^{N} .
\end{aligned}
$$

In order to deal with bounded functions, we take advantage of the invariance of the equation with respect to the change $u^{\varepsilon} \mapsto \varphi\left(u^{\varepsilon}\right)$ where $\varphi$ is, say, a $C^{1}$, Lipschitz continuous, bounded function such that $\varphi^{\prime}>0$ in $\mathbb{R}$. We still denote by $u^{\varepsilon}$ this new function.

Assuming at least that $d\left(\varepsilon \Gamma_{0}, \bar{\Gamma}_{0}\right) \rightarrow 0$ when $\varepsilon \downarrow 0$ and applying the results of Lions, Papanicolaou and Varadhan [23], we deduce that there exists a Hamiltonian $\bar{H}: \mathbb{R}^{N} \rightarrow \mathbb{R}$ such that $u^{\varepsilon}$ converges locally uniformly to $\bar{u}$ which is the unique viscosity solution of

$$
\begin{aligned}
& \bar{u}_{t}+\bar{H}(D \bar{u})=0 \quad \text { in }(0,+\infty) \times \mathbb{R}^{N}, \\
& \bar{u}(x, 0)=\bar{u}_{0}(x)=\varphi\left(d\left(x, \bar{\Gamma}_{0}\right)\right) \quad \text { in } \mathbb{R}^{N} .
\end{aligned}
$$

In order to proceed, we first remark that this local uniform convergence is enough to study the behaviour of the moving front: indeed, using the fact that $m \leqslant R(x) \leqslant M$ in $\mathbb{R}^{N}$ for some constants $m, M>0$, we have

$$
x \in \Gamma_{t} \Rightarrow m t \leqslant d\left(x, \Gamma_{0}\right) \leqslant M t .
$$

And therefore, after the scaling it is enough to control the behaviour of $u^{\varepsilon}$ in a bounded region. Moreover, coming back to the above change of variables through $\varphi$, the same argument shows that if $\varphi(s)=s$ for, say, $|s| \leqslant 1$, then the change plays no role except simplifying the arguments.

On the other hand, as we will see in the example below treating the evolution of a bounded initial front, the $\varepsilon$-transformation that we did above on $u$ has the disadvantage of discarding some information on the burnt region.

To point out the main difficulties and differences, we first assume that the set $\left\{x: d\left(x, \bar{\Gamma}_{0}\right)<0\right\}$ is nonempty and for any $t>0$, we denote by $\bar{\Gamma}_{t}$ the set $\{\bar{u}(t, \cdot)=0\}$.

If $(t / \varepsilon, x / \varepsilon) \in \Gamma_{t / \varepsilon}$ for some $t>0$, the above result yields

$$
0=\varepsilon u(t / \varepsilon, x / \varepsilon)=\bar{u}(t, x)+o(1) .
$$

Therefore $\bar{u}(t, x)=o(1)$; but in order to relate this property to $\bar{\Gamma}_{t}$ and in particular to show that $x$ is close to $\bar{\Gamma}_{t}$, we need the assumption that $\left\{d\left(\cdot, \bar{\Gamma}_{0}\right)<0\right\}$ is not empty (see the example below).

If this assumption holds, we can use the result of Ley [22, Thm. 4.2, p. 557] which implies that $d\left(x, \bar{\Gamma}_{t}\right)=o(1)$, i.e. $d\left(\varepsilon \Gamma_{t / \varepsilon}, \bar{\Gamma}_{t}\right) \rightarrow 0$ as $\varepsilon \rightarrow 0$ or equivalently $d\left(\frac{t}{T} \Gamma_{T}, \bar{\Gamma}_{t}\right) \rightarrow 0$ as $T \rightarrow+\infty$. In particular, for $t=1$, we see that $\Gamma_{T} \sim T \bar{\Gamma}_{1}$ for large $T>0$.

Next, we have to compare $T \bar{\Gamma}_{1}$ and $\bar{\Gamma}_{T}$. We first remark that, because of its definition, $\bar{\Gamma}_{0}$ is a cone, i.e. for any $a>0, a \bar{\Gamma}_{0}=\bar{\Gamma}_{0}$. Then the level set approach shows that the signs of $\bar{u}$ are independent of $\varphi$ and in particular we may choose $\varphi$ to be the identity on $\mathbb{R}$. With this choice, using the fact that $\bar{H}$ is positively homogeneous of degree 1 , one shows easily that, for any $a>0$,

$$
a \bar{u}(x / a, t / a)=\bar{u}(x, t) \quad \text { in }(0,+\infty) \times \mathbb{R}^{N},
$$

and the equality $\bar{\Gamma}_{a}=a \bar{\Gamma}_{1}$ follows immediately for any $a>0$. 
We deduce from this property that

$$
\frac{1}{T} d\left(\Gamma_{T}, \bar{\Gamma}_{T}\right)=d\left(\frac{1}{T} \Gamma_{T}, \frac{1}{T} \bar{\Gamma}_{T}\right)=d\left(\frac{1}{T} \Gamma_{T}, \bar{\Gamma}_{1}\right) \rightarrow 0
$$

as $T \rightarrow+\infty$, which ends the proof of (i).

We first prove (ii) in the case when the set $\left\{d\left(\cdot, \bar{\Gamma}_{0}\right)<0\right\}$ is not empty. If $d\left(\Gamma_{0}, \bar{\Gamma}_{0}\right)=O(\varepsilon)$, combining the maximum principle with (the easy part of) the estimates relating to convergence of $u^{\varepsilon}$ to $\bar{u}$ given in Capuzzo-Dolcetta and Ishii [11], we have

$$
\varepsilon u(x / \varepsilon, t / \varepsilon)=\bar{u}(x, t)+O(\varepsilon) .
$$

Choosing again $(x / \varepsilon, t / \varepsilon) \in \Gamma_{t / \varepsilon}$ for some $t>0$ yields $\bar{u}(x, t)=O(\varepsilon)$ and by the results of [22], we are led this time to $d\left(\varepsilon \Gamma_{t / \varepsilon}, \bar{\Gamma}_{t}\right)=O(\varepsilon)$ or equivalently, for large $T, d\left(\Gamma_{T}, \bar{\Gamma}_{T}\right)=O(1)$.

Now we turn to case (ii) when the set $\left\{x: d\left(x, \bar{\Gamma}_{0}\right)<0\right\}$ is empty; in this case, for any $t>0$, we denote by $\bar{\Gamma}_{t}$ the boundary of the set $\{\bar{u}(\cdot, t)>0\}$. To obtain the result, we apply an idea presented in Soravia [29] and using an approach by optimal control and minimum time function: let us denote by $\Omega_{0}$ the set $\left\{x: d\left(x, \Gamma_{0}\right)<0\right\}$ and let us introduce the functions $\chi^{\varepsilon}: \mathbb{R}^{N} \rightarrow \mathbb{R}$ satisfying $\chi^{\varepsilon}(x)=0$ on $\varepsilon \bar{\Omega}_{0}$ and

$$
R(x / \varepsilon)\left|D \chi^{\varepsilon}\right|=1 \quad \text { in } \mathbb{R}^{N}-\varepsilon \bar{\Omega}_{0}
$$

It is proved in [29] that, for any $t>0$, the functions $\chi^{\varepsilon}(x)-t$ and $u^{\varepsilon}(x, t)$ have the same strict signs. This can be seen rather easily here by using the representation formulas for $\chi^{\varepsilon}$ and $u^{\varepsilon}$ or by remarking that $\mathbb{1}_{\left\{\chi^{\varepsilon}(x)-t>0\right\}}-\mathbb{1}_{\left\{\chi^{\varepsilon}(x)-t \leqslant 0\right\}}$ and $\mathbb{1}_{\left\{u^{\varepsilon}>0\right\}}-\mathbb{1}_{\left\{u^{\varepsilon} \leqslant 0\right\}}$ are two lower semicontinuous viscosity solutions of [2.2) with the same initial data, and by a result of Barron and Jensen [8] (see also Barles [4] or Barles, Soner and Souganidis [5]) such a lsc solution is unique.

On the other hand, $\chi^{\varepsilon}$ converges locally uniformly in $\mathbb{R}^{N}$ to the unique viscosity solution $\bar{\chi}$ of

$$
\bar{H}(D \bar{\chi})=1 \quad \text { in } \mathbb{R}^{N}-\bar{\Gamma}_{0},
$$

with $\bar{\chi}(x)=0$ on $\bar{\Gamma}_{0}$. Therefore, if as above, $(t / \varepsilon, x / \varepsilon) \in \Gamma_{t / \varepsilon}$ for some $t>0$, then $\chi^{\varepsilon}(x)=t$ and therefore

$$
\bar{\chi}(x)-t=o(1) .
$$

In order to conclude, we remark that, if $\bar{u}$ is defined as above, then the sets $\{\bar{u}(\cdot, t) \leqslant 0\}$ and $\{\bar{\chi}(\cdot)-t \leqslant 0\}$ coincide: to prove this, it suffices to use the equality

$$
\mathbb{1}_{\left\{\chi^{\varepsilon}(x)-t>0\right\}}-\mathbb{1}_{\left\{\chi^{\varepsilon}(x)-t \leqslant 0\right\}}=\mathbb{1}_{\left\{u^{\varepsilon}>0\right\}}-\mathbb{1}_{\left\{u^{\varepsilon} \leqslant 0\right\}} \quad \text { in }[0,+\infty) \times \mathbb{R}^{N},
$$

and to pass to the limit in a suitable way.

EXAMPLES 1 . If $\Gamma_{0}$ is bounded, then it is easy to see that $\varepsilon d\left(x / \varepsilon, \Gamma_{0}\right) \rightarrow \bar{u}_{0}(x):=|x|$ and $\bar{\Gamma}_{0}$ $=\{0\}$. Since $\bar{u}_{0}$ is convex and $\bar{H}$ is only a function of $p$, Hopf's formula applies and we have (for $\varphi$ equal to the identity)

$$
\bar{u}(x, t)=\sup _{|p| \leqslant 1}[p \cdot x-t \bar{H}(p)] .
$$

But, from [23], $\bar{H}$ is convex and homogeneous of degree 1; therefore there exists some convex compact subset $K$ of $\mathbb{R}^{N}$ such that the Fenchel conjugate $\bar{H}^{*}$ of $\bar{H}$ is given by

$$
\bar{H}^{*}(q)= \begin{cases}0 & \text { if } q \in K, \\ +\infty & \text { otherwise. }\end{cases}
$$


Because of the form of $\bar{u}$, it is also clear that $\bar{u}(x, t)$ has the same behaviour as $t \bar{H}^{*}(x / t)$ and more precisely that

$$
\begin{cases}\bar{u}(t, x)=0 & \text { if } x / t \in K, \\ \bar{u}(t, x)>0 & \text { otherwise. }\end{cases}
$$

In crystalline evolution, $K$ is called a Wulff shape. As is the case here, Wulff shapes govern the asymptotic behaviour of moving fronts; we refer to Soravia [29] and references therein for a more complete discussion.

2. The second example concerns the asymptotic evolution of flat fronts, i.e. $u_{0}(x)=p \cdot x-a$ for $p \in \mathbb{R}^{N}-\{0\}$ and $a \in \mathbb{R}$. Here since the scaling just transforms $u_{0}$ into $p \cdot x$, all computations are straightforward and we have

$$
\bar{u}(t, x)=p \cdot x-t \bar{H}(p) .
$$

Therefore $\bar{\Gamma}_{t}:=\{x: p \cdot x-t \bar{H}(p)=0\}$, which means that, asymptotically, the front has normal velocity $\bar{H}(p)$.

\section{An explicit computation}

In this section, we consider in $\mathbb{R}^{2}$ a function $R$ of the form

$$
R(x, y)= \begin{cases}M & \text { if } y \in \mathbb{Z}, \\ m & \text { otherwise },\end{cases}
$$

where $m, M$ are positive constants. We have in mind that $m \ll M$.

The aim of this section is to make explicit all the quantities appearing in the previous section. Of course, $R$ is discontinuous and therefore neither the result nor the arguments of the preceding section can be applied: this is why the computations below are formal but we justify them by showing at the end of the section a "stability result" which says essentially that if we have a sequence of Lipschitz continuous $\left(R_{\varepsilon}\right)_{\varepsilon}$ which converge in a suitable sense to $R$, then the corresponding $\bar{H}_{\varepsilon}$ converge to the $\bar{H}$ we are going to compute now.

To do this computation, we come back to the equation

$$
R(x / \varepsilon, y / \varepsilon)\left|D \chi^{\varepsilon}\right|=1,
$$

which was set in the proof of Theorem 2.1 in $\mathbb{R}^{N}-\varepsilon \bar{\Omega}_{0}$. In fact, since we are only interested in $\bar{H}$, we may as well consider it in $\mathbb{R}^{N}-\{(0,0)\}$; and to compute explicitly the solution, we remark that $\chi^{\varepsilon}$ is given by $\chi^{\varepsilon}(x, y)=\varepsilon w(x / \varepsilon, y / \varepsilon)$ where the function $w$ is the solution of

$$
R(x, y)|D w|=1 \quad \text { in } \mathbb{R}^{2}-\{0\},
$$

with $w(0,0)=0$.

Formally (since $R$ is discontinuous) $w$ is given by

$$
\begin{array}{r}
w(x, y)=\inf \left\{T: \text { there exists } \gamma:[0, T] \rightarrow \mathbb{R}^{2} \text { with } \gamma(0)=(x, y),\right. \\
\qquad \gamma(T)=(0,0) \text { and }|\dot{\gamma}| \leqslant R(\gamma)\},
\end{array}
$$

where $\gamma$ is taken among all piecewise $C^{1}$ paths. 
Because of the special form of $R$, the computation of $w$ is rather easy; indeed, it is clear that optimal trajectories can be taken of the following form: a straight line from $(x, y)$ to some point $\left(x_{0}, 0\right)$ with $|\dot{\gamma}(s)|=m$ and then another horizontal line from $\left(x_{0}, 0\right)$ to $(0,0)$ with $|\dot{\gamma}(s)|=M$. An optimization with respect to $x_{0}$ yields

$$
w(x, y)= \begin{cases}\frac{\left(x^{2}+y^{2}\right)^{1 / 2}}{m} & \text { if }|y|>\frac{M}{m}|x|, \\ \frac{1}{M}\left(|x|-\frac{m}{M}|y|\right)+\frac{1}{m}\left(\left(\frac{m}{M}\right)^{2}+1\right)^{1 / 2}|y| & \text { otherwise. }\end{cases}
$$

Since $w$ is homogeneous of degree 1 , the computation of the Wulff shape $K$ is simple: indeed, the burnt region at time $t$ looks like $\{(x, y): w(x, y) \leqslant t\}$ which is nothing but $t K$ with $K=$ $\{(x, y): w(x, y) \leqslant 1\}$ because of the homogeneity of $w$. Then a rather simple optimization provides

$$
\bar{H}(p)=\sup _{q \in K}(p \cdot q)=\max \left(M\left|p_{1}\right|, m|p|\right)
$$

if $p=\left(p_{1}, \ldots, p_{N}\right)$.

It remains to consider the stability of this formula.

Proposition 3.1 Assume that $\left(R_{\varepsilon}\right)_{\varepsilon}$ is a decreasing sequence of Lipschitz continuous, periodic functions defined on $\mathbb{R}$ such that $m \leqslant R_{\varepsilon}(y) \leqslant M$ on $\mathbb{R}$, which converges pointwise to $R$. Then, for any $p \in \mathbb{R}^{2}$, the corresponding $\bar{H}_{\varepsilon}(p)$ converges to $\bar{H}(p)=\max \left(M\left|p_{1}\right|, m|p|\right)$.

Proof. We first remark that, since the sequence $\left(R_{\varepsilon}\right)_{\varepsilon}$ is decreasing, the sequence $\left(\bar{H}_{\varepsilon}\right)_{\varepsilon}$ is decreasing as well and therefore convergent because of the estimates we have on the $\bar{H}_{\varepsilon}$ 's, namely $m|p| \leqslant \bar{H}_{\varepsilon}(p) \leqslant M|p|$.

Because of the symmetries in the problems, we may assume without loss of generality that $p_{1}, p_{2} \geqslant 0$ and $|p|=1$.

The $\bar{H}_{\varepsilon}(p)$ can also be viewed as the velocities of travelling waves and it is shown in [25] that these velocities are given by the following formulas where $\alpha$ denotes the slope between the $y$-axis and the planar front, i.e. $\alpha=p_{2} / p_{1}$ :

- If

$$
\alpha>\alpha_{0}^{\varepsilon}:=\int_{0}^{1}\left(\frac{\left\|R_{\varepsilon}\right\|_{\infty}^{2}}{R_{\varepsilon}(z)^{2}}-1\right)^{1 / 2} \mathrm{~d} z
$$

then $\bar{H}_{\varepsilon}(p)$ is the unique solution $V$ of

$$
\alpha=\int_{0}^{1}\left(\left(1+\alpha^{2}\right) \frac{V^{2}}{R_{\varepsilon}(z)^{2}}-1\right)^{1 / 2} \mathrm{~d} z .
$$

- If $\alpha \leqslant \alpha_{0}^{\varepsilon}$, then $\bar{H}_{\varepsilon}(p)=\left\|R_{\varepsilon}\right\|_{\infty} /\left(1+\alpha^{2}\right)^{1 / 2}$.

By letting $\varepsilon$ go to 0 , then $\alpha_{0}^{\varepsilon} \rightarrow \alpha_{0}:=\left(M^{2} / m^{2}-1\right)^{1 / 2}$ and for $\alpha>\alpha_{0}, \bar{H}_{\varepsilon}(p)$ converges to the unique solution $V$ of

$$
\alpha=\int_{0}^{1}\left(\left(1+\alpha^{2}\right) \frac{V^{2}}{m^{2}}-1\right)^{1 / 2} \mathrm{~d} z,
$$

i.e. $V=m$. If, on the contrary, $\alpha<\alpha_{0}$, then $\bar{H}_{\varepsilon}(p)=M /\left(1+\alpha^{2}\right)^{1 / 2}$. For $p_{1}>0$ and $|p|=1$, this provides the right answer, i.e. $\bar{H}_{\varepsilon}(p)=M p_{1}$. 


\section{Existence of a line of maximal speed}

In this section, we study in a more precise way the propagation of flame fronts in the case when $R$ has lines of maximal speed and when the initial front is a graph with respect to variables which are orthogonal to these lines. Our aim is to show that, as in the previous section, the combustion is driven by these lines of maximal speed as it was driven above by $M$ for $p=(1,0)$, and to find a more accurate asymptotic behaviour of the front.

To be more specific, for $x \in \mathbb{R}^{N}$, we set $x=\left(x_{1}, x_{2}\right)$ with $x_{1} \in \mathbb{R}$ and $x_{2} \in \mathbb{R}^{N-1}$ and we assume that

$$
\begin{aligned}
& \forall n \in \mathbb{Z}^{N-1}, \quad R_{0}\left(x_{1}\right):=R\left(x_{1}, n\right)=\max _{x_{2} \in \mathbb{R}^{N-1}} R\left(x_{1}, x_{2}\right), \quad \text { and } \\
& \forall x_{2} \notin \mathbb{Z}^{N-1}, \quad \forall x_{1} \in \mathbb{R}, \quad R\left(x_{1}, x_{2}\right)<R_{0}\left(x_{1}\right) .
\end{aligned}
$$

We point out that the second part of the above assumption is purely technical; the convergence theorem below would not be altered without it.

We consider the solutions $\xi\left(t, x_{1}\right)$ of the ordinary differential equation

$$
\dot{\xi}\left(t, x_{1}\right)=R_{0}\left(\xi\left(t, x_{1}\right)\right), \quad \xi\left(0, x_{1}\right)=x_{1} .
$$

One checks easily that the solutions of (4.1) are 1-periodic in $x_{1}$ and if $T$ is given by

$$
T=\int_{0}^{1} \frac{\mathrm{d} x}{R_{0}(x)},
$$

then $\xi\left(t+T, x_{1}\right)=\xi\left(t, x_{1}\right)+1$ for any $t$ and $x_{1}$.

For the initial front $\Gamma_{0}$, we suppose that it is of the form $x_{1}=\phi\left(x_{2}\right)$, where $\phi$ is a continuous, $\mathbb{Z}^{N-1}$-periodic function and we represent it through a Lipschitz continuous initial datum $u_{0}$, strictly increasing in $x_{1}, \mathbb{Z}^{N-1}$-periodic in $x_{2}$, such that $-1 \leqslant u_{0} \leqslant 1$ in $\mathbb{R}^{N}, u_{0}\left(x_{1}, x_{2}\right)<0$ if $x_{1}<\phi\left(x_{2}\right)$, $u_{0}\left(x_{1}, x_{2}\right)>0$ if $x_{1}>\phi\left(x_{2}\right)$ and

$$
\lim _{x_{1} \rightarrow-\infty} u_{0}\left(x_{1}, x_{2}\right)=-1, \quad \lim _{x_{1} \rightarrow+\infty} u_{0}\left(x_{1}, x_{2}\right)=1, \quad \text { uniformly in } x_{2} \in \mathbb{R}^{N-1} .
$$

We still denote by $u\left(t, x_{1}, x_{2}\right)$ the solution of the Cauchy problem with initial data $u_{0}$, by $\Gamma_{t}$ the 0-level set of $u(t, \cdot, \cdot)$ at time $t$ and by $\tilde{\Gamma}_{t}$ the front driven by $R_{0}$, which is nothing but

$$
\left\{\left(\xi\left(t, x_{1}\right), x_{2}\right):\left(x_{1}, x_{2}\right) \in \Gamma_{0}\right\} .
$$

Our main result is

THEOREM 4.1 The following properties hold:

(i) The distance between $\Gamma_{t}$ and $\tilde{\Gamma}_{t}$ remains bounded.

(ii) There exists a Lipschitz continuous function $u_{\infty}\left(t, x_{1}, x_{2}\right)$, satisfying (4.3), $T$-periodic in $t$ and $\mathbb{Z}^{N-1}$-periodic in $x_{2}$, such that

$$
\lim _{t \rightarrow+\infty}\left\|u\left(t, \xi\left(t, x_{1}\right), x_{2}\right)-u_{\infty}\left(t, x_{1}, x_{2}\right)\right\|_{\infty}=0 .
$$

This result can be viewed as an asymptotic expansion of the front $\Gamma_{t}$ for large times: (i) means that the first term in this expansion is $\tilde{\Gamma}_{t}$, which justifies our above claim that the combustion is driven by 
lines of maximal speed, and (ii) provides the second term since it essentially means that $\Gamma_{t}$ differs from $\tilde{\Gamma}_{t}$ by a periodic motion: in fact, through the change $\xi\left(t, x_{1}\right)$, we essentially observe the front propagation in coordinates moving with $\tilde{\Gamma}_{t}$ and what we see is a periodic phenomenon.

Proof of Theorem 4.1] It is a variant of [25]. Following [25], one shows easily that $\left\|u_{t}\right\|_{\infty}$ and $\|D u\|_{\infty}$ are uniformly bounded and by a comparison result we also have

$$
-1 \leqslant u\left(t, x_{1}, x_{2}\right) \leqslant 1 \quad \text { in }[0,+\infty) \times \mathbb{R}^{N} .
$$

To get compactness, we simply need to be a little bit more careful and the following special lemma is devoted to this question. Moreover, (i) is also an easy consequence of this lemma.

LEMMA 4.1 We have

$$
\lim _{x_{1} \rightarrow-\infty} u\left(t, \xi\left(t, x_{1}\right), x_{2}\right)=-1, \quad \lim _{x_{1} \rightarrow+\infty} u\left(t, \xi\left(t, x_{1}\right), x_{2}\right)=+1,
$$

uniformly with respect to $\left(t, x_{2}\right) \in \mathbb{R}_{+} \times \mathbb{R}^{N-1}$.

Proof.

1. The limit when $x_{1} \rightarrow+\infty$. Here we just use the fact that $R\left(x_{1}, x_{2}\right) \leqslant R_{0}\left(x_{1}\right)$ in $\mathbb{R}^{N}$ and we are going to build a suitable, one-dimensional subsolution $\underline{u}$ of the Cauchy problem. We set $\underline{u}_{0}\left(x_{1}\right):=\min _{x_{2} \in \mathbb{R}^{N-1}} u_{0}\left(x_{1}, x_{2}\right)$. Let $\underline{u}$ be the solution of

$$
\begin{array}{ll}
\underline{u}_{t}+R_{0}\left(x_{1}\right)|D \underline{u}|=0 & \text { in }(0,+\infty) \times \mathbb{R}^{N}, \\
\underline{u}\left(x_{1}, x_{2}, 0\right)=\underline{u}_{0}\left(x_{1}\right) & \text { in } \mathbb{R}^{N} .
\end{array}
$$

Clearly, $\underline{u}$ is a subsolution to $1.2, \underline{u}_{0} \leqslant u_{0}$ in $\mathbb{R}^{N}$, therefore we have

$$
\underline{u}\left(t, x_{1}, x_{2}\right) \leqslant u\left(t, x_{1}, x_{2}\right) \quad \text { in }[0,+\infty) \times \mathbb{R}^{N} .
$$

Moreover, we can compute $\underline{u}$ explicitly by using the method of characteristics: for $\left(x_{1}, x_{2}\right) \in \mathbb{R}^{N}$ and $t \geqslant 0$, we have

$$
\underline{u}\left(t, x_{1}, x_{2}\right)=\underline{u}_{0}\left(\xi\left(-t, x_{1}\right)\right),
$$

and plugging this in the above inequality yields

$$
\underline{u}_{0}\left(x_{1}\right) \leqslant u\left(t, \xi\left(t, x_{1}\right), x_{2}\right) \quad \text { in }[0,+\infty) \times \mathbb{R}^{N} .
$$

Now the result for $x_{1} \rightarrow+\infty$ follows from the assumptions on $u_{0}$.

2. The limit when $x_{1} \rightarrow-\infty$. In this case we use the representation formula given in the Introduction, which reads here

$$
u\left(t, x_{1}, x_{2}\right)=\inf _{\gamma(t)=\left(x_{1}, x_{2}\right),|\dot{\gamma}| \leqslant R(\gamma)} u_{0}(\gamma(0)),
$$

where $\gamma$ is taken among all piecewise $C^{1}$ curves.

To estimate $u\left(t, x_{1}, x_{2}\right)$ from above we are going to use a particular path $\gamma$. Of course we may assume that $t$ is large, otherwise the behaviour just follows from the finite speed of propagation. 
Let us denote by $\left[x_{2}\right] \in \mathbb{Z}^{N-1}$ the point of $\mathbb{Z}^{N-1}$ closest to $x_{2}$ (or one of such points). We assume that $\left[x_{2}\right] \neq x_{2}$ (otherwise the argument is even easier) and we set $e=\left(\left[x_{2}\right]-x_{2}\right) /\left|\left[x_{2}\right]-x_{2}\right|$ and

$$
\gamma(s)= \begin{cases}\left(x_{1}, x_{2}\right)+m(t-s)(0, e) & \text { if } t-\bar{t} \leqslant s \leqslant t \\ \left(\xi\left(s-(t-\bar{t}), x_{1}\right),\left[x_{2}\right]\right) & \text { for } 0 \leqslant s \leqslant \bar{t}\end{cases}
$$

where $\bar{t}>0$ is defined by $x_{2}+m \bar{t} e=\left[x_{2}\right]$. It is easy to see that $\gamma$ is an admissible trajectory (i.e. $|\dot{\gamma}| \leqslant R(\gamma)$ ) and by the dynamic programming principle or the semigroup property of the equation, we have

$$
u\left(t, x_{1}, x_{2}\right) \leqslant u\left(t-\bar{t}, x_{1},\left[x_{2}\right]\right) \leqslant u_{0}\left(\xi\left(\bar{t}-t, x_{1}\right),\left[x_{2}\right]\right) .
$$

From this we deduce the inequality

$$
u\left(t, \xi\left(t, x_{1}\right), x_{2}\right) \leqslant u_{0}\left(\xi\left(\bar{t}-t, \xi\left(t, x_{1}\right)\right),\left[x_{2}\right]\right)=u_{0}\left(\xi\left(\bar{t}, x_{1}\right),\left[x_{2}\right]\right) .
$$

Since $\bar{t}$ is clearly bounded, the limit at $-\infty$ of $u\left(t, \xi\left(t, x_{1}\right), x_{2}\right)$ follows easily.

We come back to the proof of Theorem 4.1. We set

$$
v\left(t, x_{1}, x_{2}\right)=u\left(t, \xi\left(t, x_{1}\right), x_{2}\right) \quad \text { in }[0,+\infty) \times \mathbb{R}^{N} .
$$

In order to study the properties of $v$, we first notice that $\zeta\left(t, x_{1}\right):=\frac{\partial \xi}{\partial x_{1}}\left(t, x_{1}\right)$ is a solution of

$$
\dot{\zeta}=R_{0}^{\prime}(\xi) \zeta, \quad \zeta\left(x_{1}, 0\right)=1,
$$

and therefore by uniqueness for this ordinary differential equation, we have

$$
\zeta\left(t, x_{1}\right)=\frac{\dot{\xi}\left(t, x_{1}\right)}{R_{0}\left(x_{1}\right)}=\frac{R\left(\xi\left(t, x_{1}\right)\right)}{R_{0}\left(x_{1}\right)} .
$$

In particular, $m M^{-1} \leqslant \zeta\left(t, x_{1}\right) \leqslant M m^{-1}$ for any $x_{1} \in \mathbb{R}$ and $t \geqslant 0$.

Because $\left(t, x_{1}\right) \mapsto \xi\left(t, x_{1}\right)$ is smooth, the chain rule applies and $v$ is a viscosity solution in $[0,+\infty) \times \mathbb{R}^{N}$ of the equation

$$
v_{t}+R\left(t, \xi\left(t, x_{1}\right), x_{2}\right) \sqrt{\frac{R_{0}\left(x_{1}\right)^{2}}{R_{0}\left(\xi\left(t, x_{1}\right)\right)^{2}} v_{x_{1}}^{2}+v_{x_{2}}^{2}}-R_{0}\left(x_{1}\right) v_{x_{1}}=0 .
$$

We make the following remarks which will permit us to end the proof:

- Because of the preceding remark and Lemma 4.1, the family $(v(t, \cdot, \cdot))_{t}$ is relatively compact in $C\left(\mathbb{R}^{N}\right)$.

- Because of the definition of $R_{0}$, we see that, for all $(x, y) \in \mathbb{R} \times \mathbb{Z}^{N-1}$, the function $t \mapsto$ $v\left(t, x_{1}, x_{2}\right)$ is nonincreasing: indeed, on this set the nonlinearity of the equation is positive. Hence, on $\mathbb{R} \times \mathbb{Z}^{N-1}, v\left(t, x_{1}, x_{2}\right)$ converges to a steady state on this set; this steady state is strictly between -1 and 1 due to the preceding remarks.

- On the complement of this set, i.e. on $\mathbb{R}^{N}-\left(\mathbb{R} \times \mathbb{Z}^{N-1}\right)$, the function $x_{1} \mapsto \tanh \left(x_{1}\right)$ is a strict subsolution to 4.5).

Finally, the Hamiltonian of equation (4.5) being periodic in $t$ and $x_{2}$, the arguments of the proof of Theorem 1 in [25] apply, yielding the convergence of $v(t, \cdot, \cdot)$ to a solution of (4.5) which is also periodic in $t$ and $x_{2}$. Thus the proof is complete. 
REMARK 4.1 The role played by the straight line of maximum speed is obvious in view of formula $(1.2)$ : it is simply a geodesic for the distance function

$$
\delta(X, Y)=\inf _{\gamma(0)=X, \gamma(t)=Y} \int_{0}^{t} \frac{|\dot{\gamma}(s)|^{2}}{R(\gamma)^{2}} \mathrm{~d} s .
$$

As a consequence we could generalize Theorem 4.1 by removing the periodicity of $R$ in $x_{1}$ and by only keeping the assumption of a line of maximal speed. The outcome is then that the dynamics of the solutions of the whole equation 1.2 is driven by the dynamics of $\dot{\xi}=R_{0}(\xi)$.

\section{Time-asymptotic behaviour in two space dimensions}

In this section, we assume $N=2$ and we consider a vector $p \in \mathbb{Z}^{2}$; in order to model a motion whose general direction of propagation is the vector $p$, we impose an initial data $u_{0}$ for (1.2) of the form

$$
u_{0}(x)=p \cdot x+v_{0}(x)
$$

where $v_{0}$ is a bounded, continuous, $\mathbb{Z}^{2}$-periodic function.

We write the solution $u$ of $(1.2)$ associated to this initial data in the form

$$
u(t, x)=p \cdot x-\bar{H}(p) t+v(t, x),
$$

where $\bar{H}(p)$ is the homogenized Hamiltonian of Section 1. The function $v$ is $\mathbb{Z}^{2}$-periodic in $x$ and solves

$$
\begin{aligned}
v_{t}+R(x)|D v+p| & =\bar{H}(p) & & \text { in }(0,+\infty) \times \mathbb{R}^{2}, \\
v(0, x) & =v_{0}(x) & & \text { in } \mathbb{R}^{2} .
\end{aligned}
$$

It is well known that equation 5.3 generates a nonlinear semigroup $\tilde{\mathcal{S}}(t)$ that is weakly contracting in the space $\mathcal{C}_{\text {per }}$ of continuous $\mathbb{Z}^{2}$-periodic functions, i.e.

$$
\forall t>0, \forall\left(u_{0}, v_{0}\right) \in \mathcal{C}_{\text {per }}, \quad\left\|\tilde{\mathcal{S}}(t) u_{0}-\tilde{\mathcal{S}}(t) v_{0}\right\|_{\infty} \leqslant\left\|u_{0}-v_{0}\right\|_{\infty} .
$$

Once and for all, we choose a $\mathbb{Z}^{2}$-periodic solution $\phi$ of the equation

$$
R(x)|D \phi+p|=\bar{H}(p) \quad \text { in } \mathbb{R}^{2} .
$$

We may trap $v_{0}$ between $\phi-C$ and $\phi+C$, where $C>0$ is a large enough constant, and then a comparison result for viscosity solutions implies

$$
\forall t>0, \quad \phi(x)-C \leqslant v(t, x) \leqslant \phi(x)+C .
$$

Moreover, since we may assume, without loss of generality (see [25]), $v_{0}$ to be Lipschitz continuous, we also have a uniform bound for $v_{t}$ and $D v$. Hence the orbit $\tilde{\mathcal{S}}(t) v_{0}$ is relatively compact in $\mathcal{C}_{\text {per }}$. This allows us to ask whether we may have a more precise result. The answer is given by

ThEOREM 5.1 There exists $T(p)>0$ such that, for every $v_{0} \in \mathcal{C}_{\text {per }}$, there is a function $v_{\infty}(t, x)$ which is $T(p)$-periodic in time and such that

$$
\lim _{t \rightarrow+\infty}\left\|\tilde{\mathcal{S}}(t) v_{0}-v_{\infty}(t, .)\right\|_{\infty}=0 .
$$


Outline of the proof. First, because of the periodicity, we may assume that the problem is posed on the 2-dimensional torus $\mathbf{T}^{2}$. The method of proof follows closely the scheme used in [28] which can, roughly speaking, be described in the following way: in order to study the asymptotic behaviour of $v$ in $\mathbb{R}^{2}$, it is enough to study it on the so-called Aubry-Mather set $\mathcal{M}_{0} \subset \mathbb{R}^{2}$ (see the definition below). Indeed, Theorem 4.8 in [28] says that two solutions which coincide on $\mathcal{M}_{0}$ coincide everywhere and, in particular, a solution which is time-periodic on $\mathcal{M}_{0}$ is time-periodic everywhere. This implies that the convergence to a time-periodic solution on $\mathcal{M}_{0}$ means such a convergence everywhere.

Next the study of the behaviour of the solutions on $\mathcal{M}_{0}$ is done in two steps: the first one is independent of the space dimension, and aims at proving a time invariance property of the trajectories of $\tilde{\mathcal{S}}(t)$ on the Aubry-Mather set associated to the geodesics. In [28], the strict convexity of the Hamiltonian implies that, if $\phi$ solves (5.5) and $\psi \in \omega\left(u_{0}\right)$, then $\psi-\phi$ does not depend on $\psi$ on the Aubry-Mather set. A relaxed semi-limit argument concludes the proof.

However, strict convexity is missing here. The only way we have to replace it, is a better knowledge of the dynamics of the extremals, which is very complicated in space dimension larger than 2, but available in 2-D: see Bangert [2]. The core of the argument is a Poincaré-Bendixson type argument: two trajectories cannot cross, hence-at least in the case of a rational rotation numberthe Poincaré map is increasing.

\subsection{Monotonicity}

If $\phi$ solves 5.5 then it is also a viscosity solution to

$$
F(X, D \phi)=0 \quad \text { with } \quad F(x, q):=R(x)^{2}|q+p|^{2}-\bar{H}(p)^{2} .
$$

The Hamiltonian $F$ is, this time, strictly convex with respect to $q$. Recall that if $L$ is the Legendre transform of $F$ with respect to $q$, then for all $\phi$ solving 5.8$]$ we have

$$
\forall t>0, \quad \phi(x)=\inf _{\gamma \in C^{1}\left([0, t], \mathbf{T}^{2}\right), \gamma(t)=x}\left(\phi(\gamma(0))+\int_{0}^{t} L(\gamma(s), \dot{\gamma}(s)) \mathrm{d} s\right) .
$$

By results of Fathi [17], $\phi$ is $C^{1}$ on the Aubry-Mather set $\mathcal{M}_{0}$ and it is classical that the geodesic problem in (5.9) generates in fact a nonlinear backward semigroup $\mathcal{T}_{\phi}$ on

$$
\mathcal{M}_{\phi}=\{\gamma(0): \gamma \text { extremal in } 5.9\}
$$

Indeed, this dynamical system is given by the associated Hamiltonian system: set $x(t):=\gamma(t)$ and $q(t)=D \phi(\gamma(t))$; then the solution $(x(t), q(t))$ of the $F$-Hamiltonian system is related to the geodesics through $q(t)=L_{q}(\gamma(t), \dot{\gamma}(t))$.

Let $\mathcal{H}$ be the set of solutions of $\left[5.9\right.$. The Aubry-Mather set $\mathcal{M}_{0}$ can be defined by the following algorithm given in [28]:

$$
\mathcal{M}_{1}(\phi)=\bigcup_{x \in \mathcal{M}_{\phi}} \alpha_{\mathcal{T}_{\phi}}(x), \quad \mathcal{M}_{0}(\phi)=\bigcup_{x \in \mathcal{M}_{1}} \omega_{\mathcal{T}_{\phi}}(x), \quad \mathcal{M}_{0}=\bigcap_{\phi \in \mathcal{H}} \mathcal{M}_{0}(\phi) .
$$

As is classical, $\alpha_{\mathcal{T}}(x)$ (resp. $\left.\omega_{\mathcal{T}}(x)\right)$ is the $\alpha$-limit (resp. $\omega$-limit) set of the point $x$ under the action of the semigroup $\mathcal{T}$. As already mentioned at the beginning of the proof but in a slightly different context, two elements of $\mathcal{H}$ coinciding on $\mathcal{M}_{0}$ coincide on $\mathbf{T}^{2}$. 
The first ingredient is

Lemma 5.1 (Dynamic Programming Principle) Let $\gamma$ be an extremal to 5.9 which is defined on $[0, T]$. We set

$$
\tilde{\gamma}(t)=\gamma\left(\frac{t}{2 \bar{H}(p)}\right) .
$$

Then the function $t \mapsto v(t, \tilde{\gamma}(t))-\phi(\tilde{\gamma}(t))$ is nonincreasing on [0,T].

Proof. We first give a formal proof and then justify it. If everything is smooth, using the fact that $\dot{\gamma}=F_{q}(\gamma, D \phi(\gamma))$, we have

$$
\begin{aligned}
\frac{\mathrm{d}}{\mathrm{d} t}(v-\phi)(t, \tilde{\gamma}(t)) & =v_{t}+D(v-\phi) \cdot \dot{\tilde{\gamma}}(t) \\
& =v_{t}+\frac{1}{2 \bar{H}(p)} D(v-\phi) \cdot F_{q}(\tilde{\gamma}, D \phi(\tilde{\gamma})) \\
& =-R(\tilde{\gamma}(t))|D v+p|+\bar{H}(p)+\frac{R(\tilde{\gamma}(t))^{2}}{\bar{H}(p)}(D \phi+p) \cdot D(v-\phi) .
\end{aligned}
$$

But $R(x)|D \phi(x)+p|=\bar{H}(p)$ and this last expression can be rewritten as

$$
-R(\tilde{\gamma}(t))\left[|D v+p|-\frac{(D \phi+p)}{|D \phi+p|} \cdot D(v-\phi)\right]+\bar{H}(p) ;
$$

by the convexity of the norm, we finally have

$$
\frac{\mathrm{d}}{\mathrm{d} t}(v-\phi)(t, \tilde{\gamma}(t)) \leqslant-R(\tilde{\gamma}(t))|D \phi+p|+\bar{H}(p)=0 .
$$

Let us justify this formal proof. Because $\phi$ is differentiable at every point $\tilde{\gamma}(t)$ with $t \in] 0, T$, the derivative $\frac{\mathrm{d}}{\mathrm{d} t} \phi(\tilde{\gamma}(t))$ exists. Hence we only have to care about $\frac{\mathrm{d}}{\mathrm{d} t} v(t, \tilde{\gamma}(t))$. Here, the argument is classical: we regularize $v$ into $v^{\varepsilon}=\delta_{\varepsilon} * v$ where $\left(\delta_{\varepsilon}\right)_{\varepsilon}$ is a sequence of approximations of unity; from Jensen's inequality, we have

$$
\left|D\left(\delta_{\varepsilon} * v\right)+p\right| \leqslant \delta_{\varepsilon} *|D v+p|,
$$

and an integration between 0 and $t \in[0, T]$ allows the passage to the limit as $\varepsilon \rightarrow 0$.

REMARK This proof can also be done without differentiating the equation using the usual form of the Dynamic Programming Principle-equivalent here to the semigroup property of $\tilde{\mathcal{S}}$ - which yields for $v$ and $\phi$ the following properties: since $\gamma$ is an extremal for $[5.9$, we have, for $0 \leqslant t \leqslant$ $s \leqslant T$,

$$
\phi(\gamma(s))=\phi(\gamma(t))+\int_{t}^{s} L(\gamma(\tau), \dot{\gamma}(\tau)) \mathrm{d} \tau .
$$

The Lagrangian $L$ is given by

$$
L(x, q)=\frac{|q|^{2}}{4 R(x)^{2}}+\bar{H}(p)^{2}-q \cdot p
$$

and since $\gamma$ is a solution of the $\operatorname{ODE} \dot{\gamma}(\tau)=2 R(\gamma(\tau))^{2}(D \phi(\gamma(\tau))+p)$ while $\phi$ solves $[5.5)$, this can be rewritten as

$$
\phi(\gamma(s))=\phi(\gamma(t))+2 \bar{H}(p)^{2}(s-t)-p \cdot(\gamma(s)-\gamma(t)) .
$$


On the other hand, again because of 5.5 , we have $|\dot{\gamma}(\tau)| \leqslant 2 R(\gamma(\tau)) \bar{H}(p)$ and therefore $|\dot{\tilde{\gamma}}(\tau)| \leqslant$ $R(\tilde{\gamma}(\tau))$. Hence, the Dynamic Programming Principle for $u$ yields

$$
u(s, \tilde{\gamma}(s)) \leqslant u(t, \tilde{\gamma}(t)),
$$

while changing $\gamma$ into $\tilde{\gamma}$ in the property satisfied by $\phi$ yields

$$
\phi(\tilde{\gamma}(s))=\phi(\tilde{\gamma}(t))+\bar{H}(p)(s-t)-p \cdot(\tilde{\gamma}(s)-\tilde{\gamma}(t)) .
$$

Subtracting them we obtain

$$
u(s, \tilde{\gamma}(s))-p \cdot \tilde{\gamma}(s)+\bar{H}(p) s-\phi(\tilde{\gamma}(s)) \leqslant u(\tilde{\gamma}(t))-p \cdot \tilde{\gamma}(t)-\bar{H}(p) t-\phi(\tilde{\gamma}(t)),
$$

which, according to the definition of $v$, is exactly what we wanted to prove.

A consequence is an analogue of Corollary 3.2 in [28].

PROPOSITION 5.1 Let $\gamma$ be an orbit of $\mathcal{T}_{\phi}$ in $\mathcal{M}_{0}$, and $\tilde{\gamma}$ defined by 5.11 . Then, if $\psi \in \omega_{\tilde{\mathcal{S}}}\left(u_{0}\right)$, the function

$$
t \mapsto \tilde{\mathcal{S}}(t) \psi-\phi
$$

is constant along $\tilde{\gamma}$.

Proof. See [28], Corollary 3.2.

Let us pause here to examine a consequence of the above proposition, on an equation given by Barles and Souganidis [6] as a counter-example to convergence to steady solutions in the case of non-strictly convex Hamiltonians:

$$
v_{t}+\left|v_{x}+1\right|-1=0, \quad x \in \mathbf{T}^{2} .
$$

The global extremals are

$$
\gamma(t)=x+t, \quad x \in \mathbf{T}^{1} .
$$

Proposition 5.1 implies that any element of $\omega\left(u_{0}\right)$ is constant along an extremal. Let us check it directly: the function $u(t, x)=v(t, x+t)$ solves

$$
v_{t}+\left|v_{x}+1\right|-v_{x}-1=0, \quad x \in \mathbf{T}^{2},
$$

which is clearly seen to converge monotonically.

\subsection{Convergence}

A large part of the study below is well known (Morse [24], Hedlund [21] for instance). An abstract setting, designed to encompass very different settings, such as geodesics, twist diffeomorphisms and extremals for the Frenkel-Kontorova model, and providing further properties, is given in [2].

The main idea is that the global trajectories of $\mathcal{T}_{\phi}$ will have some periodicity in a direction. This can be seen by a Poincaré-Bendixson type argument, that we provide for the reader's convenience.

Also, for convenience again, equation 5.3 will be assumed to be posed on $\mathbb{R}^{2}$ with periodic conditions in both directions. Also, we will assume without loss of generality that $p$ is not parallel to the vector $e_{2}$. 
First, we introduce a few definitions. A geodesic segment between two points $x$ and $y$ is a path $(\gamma(s))_{0 \leqslant s \leqslant t}$ which minimizes the action

$$
\mathcal{A}(x, y ; \gamma):=\int_{0}^{t} \frac{|\dot{\gamma}(s)|^{2}}{R(\gamma(s))^{2}} \mathrm{~d} s,
$$

among all piecewise $C^{1}$ curves $\gamma$ such that $\gamma(0)=x$ and $\gamma(t)=y$. A minimal geodesic segment is a geodesic segment such that $|\dot{\gamma}|=R(\gamma)$.

LEMMA 5.2 A global orbit of $\mathcal{T}_{\phi}$ is a minimal geodesic.

Proof. Let $\tilde{\gamma}$ be defined as in Lemma 5.1. Since $\phi$ is a solution of (5.5), the function $t \mapsto p \cdot \gamma(t)-$ $t \bar{H}(p)+\phi(\gamma(t))$ is constant; then, because $\phi$ is differentiable at all points of the orbit, we have

$$
p \cdot \dot{\tilde{\gamma}}(t)-\bar{H}(p)+\dot{\tilde{\gamma}} \cdot D \phi(\tilde{\gamma})=0
$$

which yields

$$
\dot{\tilde{\gamma}} \cdot(D \phi(\gamma)+p)=\bar{H}(p) \text {. }
$$

Next we use the fact that

$$
2 R(\gamma)^{2}(D \phi(\tilde{\gamma})+p)=\dot{\gamma}=2 \bar{H}(p) \dot{\tilde{\gamma}}(t)
$$

which yields $|\dot{\tilde{\gamma}}|=R(\tilde{\gamma})$.

In order to use a Poincaré-Bendixson argument, we first need a global section for the geodesic flow. This is not so easy as in differential equations, since a global geodesic satisfies an ODE whose vector field is not defined everywhere-in fact, only almost everywhere. We use the following two properties of minimal geodesics, which are basic to the theory of [2].

TheOREM 5.2 (Hedlund [21]) Two minimal geodesics may only cross once.

Then we construct a global section as follows: choose $x_{0}=\left(x_{10}, x_{20}\right) \in[0,1]^{2}$ which is an absolute minimum for the function

$$
x=\left(x_{1}, x_{2}\right) \in[0,1]^{2} \mapsto \inf _{\gamma} \mathcal{A}(x, x+(0,1) ; \gamma)
$$

where the infimum is taken among all piecewise $C^{1}$ functions such that $|\dot{\gamma}|=R(\gamma)$, having $x$ and $x+(0,1)$ as end points. Let $\tilde{\gamma}_{0}$ be the curve corresponding to $\left(x_{10}, x_{20}\right)$.

Theorem 5.3 (Busemann and Pedersen [9]) Set $\gamma_{0}=\left\{\tilde{\gamma}_{0}+(0, j): j \in \mathbb{Z}\right\}$. Then $\gamma_{0}$ is a global minimal geodesic.

It is worth mentioning here a generalization of this theorem in spatial dimensions $\geqslant 3$ in [10], where geodesic lines are replaced by minimal surfaces.

LEMMA 5.3 A global orbit of $\mathcal{T}_{\phi}$ intersects $\gamma_{0}$ once and only once.

Proof. Let $(\gamma(s))_{s \in \mathbb{R}}$ be such an orbit; set $x=\gamma(0)$ and look at the relation

$$
p \cdot \gamma(s)+\phi(\gamma(s))=p \cdot \gamma(0)+\phi(x)+s \bar{H}(p)+\int_{0}^{s} \frac{|\dot{\gamma}(\sigma)|^{2}}{4 R(\gamma(\sigma))^{2}} \mathrm{~d} \sigma ;
$$


because $\phi$ is bounded we have

$$
\lim _{s \rightarrow \pm \infty} p \cdot \gamma(s)= \pm \infty
$$

Hence-recall that $p$ is not parallel to $e_{2}$ - there is at least one point of intersection of $\gamma$ and $\gamma_{0}$. Uniqueness follows from the last three results.

Proof of Theorem 5.1. In two steps: convergence on $\mathcal{M}_{0}$ first, then outside $\mathcal{M}_{0}$.

1. Convergence on $\mathcal{M}_{0}$. Starting from an orbit of $\mathcal{M}_{0}$, we may define a homeomorphism $h$ of $\gamma_{0}$ as follows: if $x \in \mathcal{M}_{0} \cap \gamma_{0}$, let $y_{x}$ be the only intersection point of the orbit $\left(\mathcal{T}_{\phi}(t) x\right)_{t \in \mathbb{R}}$ and the line $e_{1}+\gamma_{0}$. Then set

$$
h(x)=y_{x}-e_{1} .
$$

This construction can be done for all points of $\mathcal{M}_{0} \cap \gamma_{0}$; hence we may define-by taking the values modulo $1-$ a homeomorphism $h$ of a part of the circle. Let $\alpha$ be its rotation number.

Case 1: $\alpha=0$. Then $h$ has a fixed point. Because two extremals cannot intersect, the sequence of iterations $h^{n}(x)$ is monotone for every $x \in \mathcal{M}_{0}(\phi)$; hence $\mathcal{M}_{0}$ is made up of periodic orbits, i.e. orbits that have the same period as the network. Let us compute the time period $T$ of $\tilde{\mathcal{S}}(t) \psi$ on $\mathcal{M}_{0}$. To do that, it is equivalent to compute the time period of the orbits. Let $\gamma$ be an orbit of $\mathcal{M}_{0}$. If $T^{\prime}$ is the time period of the orbits then we have

$$
\gamma\left(T^{\prime}\right)=\gamma(0)+e_{1}, \quad \dot{\gamma}=2 R(\gamma)^{2}(D \phi(\gamma)+p),
$$

which yields, after multiplication by $D \phi(\gamma)+p$,

$$
\frac{d}{d t}(\phi(\gamma)+p \cdot \gamma)=2 R(\gamma)^{2}|D \phi(\gamma)+p|=2 \bar{H}(p)^{2} .
$$

Integration between 0 and $T^{\prime}$ yields, by 5.17

$$
T^{\prime}=\frac{p_{1}}{2 \bar{H}(p)^{2}}, \quad \text { hence } \quad T=\frac{p_{1}}{\bar{H}(p)} .
$$

This implies that all orbits in $\mathcal{M}_{0}$ have the same time period $T^{\prime}$. Due to the Dynamic Programming Principle (Lemma 5.1), the function $u(t, \cdot)$ becomes $T^{\prime}$-periodic in $t$ on $\mathcal{M}_{0}$ as time goes to $+\infty$.

2. Convergence outside $\mathcal{M}_{0}$. The argument is more routine and will only be sketched. The flow $\tilde{\mathcal{S}}$ generates a bounded semiflow on $\mathcal{C}_{\text {per }}$; we may define the $\omega$-limit set of an initial data $v_{0}$ in the usual way, and denote it by $\omega\left(v_{0}\right)$. Then the pointwise relaxed semi-limits

$$
\underline{v}_{\infty}(t, x):=\limsup _{n \rightarrow+\infty} \tilde{\mathcal{S}}\left(t+n T^{\prime}\right) v_{0}(x), \quad \bar{v}_{\infty}(t, x)=\liminf _{n \rightarrow+\infty} \tilde{\mathcal{S}}\left(t+n T^{\prime}\right) v_{0}(x)
$$

converge to a $T^{\prime}$ time-periodic subsolution (resp. a $T^{\prime}$ time-periodic supersolution) of the equation in $v$ :

$$
\tilde{\mathcal{S}}\left(T^{\prime}\right) v=v
$$

coinciding on $\mathcal{M}_{0}$. The upshot is that $\tilde{\mathcal{S}}\left(T^{\prime}\right) \underline{v}_{\infty}(0, x)$ and $\tilde{\mathcal{S}}\left(T^{\prime}\right) \bar{v}_{\infty}(0, x)$ coincide on $\mathcal{M}_{0}$; for this we argue exactly as in the proofs of Theorems 1 and 3 in [28] for Hamilton-Jacobi equations with strictly convex Hamiltonians, the only difference is that here we should use formula (1.3) instead of the Lax-Oleĭnik formula for strictly convex Hamiltonians. 
Hence we have constructed two $n T^{\prime}$ time-periodic solutions of 5.3 coinciding on $\mathcal{M}_{0}$ and between which the whole set $\omega\left(v_{0}\right)$ lies. On the other hand, still because of the Dynamic Programming Principle, the set $\mathcal{M}_{0}$ is a uniqueness set: namely, two $n T^{\prime}$ time-periodic solutions of (5.3) coinciding on $\mathcal{M}_{0}$ coincide everywhere; see once again [28] or [19] for more details.

Case 2: $\alpha \neq 0$. The orbit $\gamma$ has then an asymptotic slope $q(\alpha)$. It suffices therefore to perform a rotation $\rho_{-\alpha}$ of angle $-2 \pi q(\alpha)$. The Hamiltonian $R(x)|D u+p|$ is replaced by $R\left(\rho_{\alpha} x\right)\left|D u+\rho_{-\alpha} p\right|$.

Let us end this paper by giving two examples related to combustion.

1. Combustion enhancement. As is stated in Sections 2 and 3, the presence of lines of maximal speed is enough for the velocity of the front to adjust to the burning velocity on these lines. In practice, this line of maximal speed models a copper wire that is placed in the solid medium in order to accelerate the combustion, but is not of zero thickness. In our context, we may be more explicit: let us consider a front propagating orthogonally to two parallel straight line enclosing an area of large speed.

THEOREM 5.4 Assume the existence of $\delta \in] 0,1[$ and $A>1$ such that

$$
\begin{aligned}
& \forall x \in \mathbb{R}, \forall y \in[0, \delta], \quad R(x, y) \geqslant A, \\
& \forall x \in \mathbb{R}, \forall y \in[2 \delta, 1], \quad R(x, y) \leqslant 1 .
\end{aligned}
$$

Then, if $A$ is large enough, we have $T\left(e_{1}\right)=\bar{H}\left(e_{1}\right)^{-1}$.

We simply notice that the global orbits of $\mathcal{T}_{\phi}$ are enclosed in the strips $S_{n}=\mathbb{R} \times[n, n+\delta]$, which implies that $\alpha=0$. The time period tends to $A^{-1}$.

2. Combustion inhibition. It occurs in certain applications that some parts of the solid propellants are prevented from burning. This can be modelled by putting the burning rate $R$ to 0 on these parts. Assume therefore that

$$
\begin{array}{ll}
\forall x \in \mathbb{R}, \forall n \in \mathbb{N}, & R(x, \pm n)=0, \\
\forall x \in \mathbb{R}, \forall y \notin \mathbb{Z}, & R(x, y)>0 .
\end{array}
$$

Let us first notice the existence of a homogenized Hamiltonian $\bar{H}(p)$ : indeed, we may pass to the limit as $\varepsilon \rightarrow 0$ in the sequence of problems

$$
(R(x, y)+\varepsilon)|D u+p|=H^{e}(p), \quad u \text { 1-periodic in } \mathbb{R}^{2} .
$$

Because we have $H^{\varepsilon}(p) \leqslant\|R\|_{\infty}+\varepsilon$, the corresponding sequence of solutions $\left(\phi^{\varepsilon}\right)_{\varepsilon}$, normalized so that $\phi^{\varepsilon}(0)=0$, converges locally uniformly. This yields the existence of a solution to the homogenized problem. Also notice that $\bar{H}(p)>0$. Once again the global orbits of $\mathcal{T}_{\phi}$ are enclosed in the strips $\mathbb{R} \times] n, n+1[$. If the initial front is the $y$-axis, the front will converge, at least on $\left.\bigcup_{n \in \mathbb{Z}} \mathbb{R} \times\right] n, n+1\left[\right.$, to periodic fronts of period $\bar{H}\left(e_{1}\right)^{-1}$.

\section{REFERENCES}

1. Aronson, D. G. \& Weinberger, H. F. Multidimensional nonlinear diffusion arising in population genetics. Adv. in Math. 30 (1978), 33-76. MR 80a:35013 Zbl 0407.92014

2. BAngert, V. Mather sets for twist maps and geodesics on tori. Dynamics Reported 1, Wiley (1988), 1-56. MR 90a:58145 Zbl 0664.53021 
3. BARLES, G. Remark on a flame propagation model. Rapport INRIA, 464 (1985).

4. BARLES, G. Discontinuous viscosity solutions of first order Hamilton-Jacobi equations: A guided visit. Nonlinear Anal. 20 (1993), 1123-1134. MR 94d:49047|Zbl 0816.35081

5. Barles, G., Soner, H. M. \& Souganidis, P. E. Front propagation and phase field theory. SIAM J. Control Optim. 31 (1993), 439-469. MR 94c:35005 Zbl 0785.35049

6. BARles, G. \& Souganidis, P. E. On the large time behaviour of solutions of Hamilton-Jacobi equations. SIAM J. Math. Anal. 31 (2000), 925-939. MR 2002b:49056 Zbl 0960.70015

7. Barles, G. \& Souganidis, P. E. Some counterexamples on the asymptotic behaviour of the solutions of Hamilton-Jacobi equations. C. R. Acad. Sci. Paris Sér. I Math. 330 (2000), 963-968. MR 2002e:35048 Zbl 0954.35046

8. Barron, N. \& Jensen, R. Semicontinuous viscosity solutions for Hamilton-Jacobi equations with convex Hamiltonians. Comm. Partial Differential Equations 15 (1990), 1713-1742. MR 91h:35069 Zbl 0732.35014

9. Busemann, H. \& Pedersen, F. P. Tori with one-parameter groups of motions. Math. Scand. 3 (1955), 209-220. MR 17,999c Zbl 0068.36405

10. Caffarelli, L. A. \& De la Llave, R. Planelike minimizers in periodic media. Comm. Pure Appl. Math. 54 (2001), 1403-1441. MR 2002j:49003

11. CAPUZzo-DolcettA, I. \& Ishi, I. On the rate of convergence in homogenization of Hamilton-Jacobi equations. International Conference on Differential Equations (Berlin, 1999), B. Fiedler et al. (eds.), Vol. 1, World Sci. (2000), 595-599. MR 2002j:35026 Zbl 0968.49019

12. Chen, Y.-G., Giga, Y., \& Goto, S. Uniqueness and existence of viscosity solutions of generalized mean curvature flow equations. J. Differential Geom. 33 (1991), 749-786. MR 93a:35093ZZbl 0696.35087

13. Concordel, M. C. Periodic homogenization of Hamilton-Jacobi equations: Additive eigenvalues and variational formula. Indiana Univ. Math. J. 45 (1996), 1095-1117. MR 98d:35018 Zbl 0871.49025

14. Concordel, M. C. Periodic homogenization of Hamilton-Jacobi equations: II: Eikonal equations. Proc. Roy. Soc. Edinburgh Sect. A 127 (1997), 665-689. MR 2000c:35012 Zbl 0883.35010

15. Evans, L. C. \& Gomes, D. Effective Hamiltonians and averaging for Hamiltonian dynamics. I. Arch. Rational Mech. Anal. 157 (2001), 1-33. MR 2002b:37099|Zbl 0986.37056

16. Evans, L. C. \& Spruck, J. Motion of level sets by mean curvature I. J. Differential Geom. 33 (1991), 635-681. MR 92h:35097/Zbl 0726.53029

17. Fathi, A. Théorème KAM faible et théorie de Mather sur les systèmes lagrangiens. C. R. Acad. Sci. Paris Sér. I 324 (1997), 1043-1046. MR 98g:58151|Zbl 0885.58022

18. FAthi, A. Solutions KAM faibles conjuguées et barrières de Peierls. C. R. Acad. Sci. Paris Sér. I Math. 325 (1997), 649-652. MR 99b:58209 Zbl 0943.37031

19. Fathi, A. Sur la convergence du semi-groupe de Lax-Oleinik. C. R. Acad. Sci. Paris Sér. I Math. 327 (1998), 267-270. MR 2000a:37058 Zbl 01235022

20. FAthi, A. \& MAther, J. N. Failure of convergence of the Lax-Oleinik semi-group in the timedependent case. Bull. Soc. Math. France 128 (2000), 473-483. MR 2001i:35023 Zbl 0989.37035

21. Hedlund, G. A. Geodesics on a two-dimensional Riemannian manifold with periodic coefficients. Ann. of Math. 33 (1932), 719-739. Zbl 0006.32601

22. LEY, O. Lower-bound gradient estimates for first-order Hamilton-Jacobi equations and applications to the regularity of propagating fronts. Adv. Differential Equations 6 (2002), 547-576. MR 2002e:35050 Zbl 01700792

23. Lions, P.-L., Papanicolaou, G. \& Varadhan, S. R. S. Unpublished preprint.

24. Morse, M. A fundamental class of geodesics on any closed surface of genus greater than one. Trans. Amer. Math. Soc. 22 (1921), 25-60. MR 1501263 
25. NAMAh, G. \& RoQUejoffre, J.-M. The "hump" effect in solid propellant combustion. Interfaces Free Bound. 2 (2000), 449-467. MR 2001j:80006 Zbl 0967.35156

26. NAmah, G. \& Roquejoffre, J.-M. Remarks on the long time behaviour of the solutions of Hamilton-Jacobi equations. Comm. Partial Differential Equations 24 (1999), 883-893. MR 2000j:35034 Zbl 0924.35028

27. Osher, S. \& Sethian, J. A. Fronts moving with curvature dependent speed: algorithms based on Hamilton-Jacobi formulations. J. Comput. Phys. 79 (1988), 12-49. MR 89h:80012 Zbl 0659.65132

28. RoqueJoffre, J.-M. Convergence to steady states or periodic solutions in a class of Hamilton-Jacobi equations. J. Math. Pures Appl. 80 (2001), 85-104. MR 2001k:35053 Zbl 0979.35033

29. Soravia, P. Generalized motion of a front propagating along its normal direction: A differential games approach. Nonlinear Anal. 22 (1994), 1247-1262. MR 96c:49049 Zbl 0814.35140 